\title{
Mobility Management for NGN WiMAX: Specification and Implementation
}

\author{
Pedro Neves ${ }^{1}$, Ricardo Matos ${ }^{2}$, Bruno Sousa ${ }^{3}$, Giada Landi ${ }^{4}$, Susana Sargento ${ }^{2}$, Kostas \\ Pentikousis $^{5}$, Marilia Curado ${ }^{3}$, Esa Piri ${ }^{5}$ \\ ${ }^{1}$ Portugal Telecom Inovação, pedro-m-neves@ptinovacao.pt, Aveiro, Portugal \\ ${ }^{2}$ University of Aveiro, \{ricardo.matos, susana\}@ua.pt, Aveiro, Portugal \\ ${ }^{3}$ University of Coimbra, \{bmsousa, marilia\}@dei.uc.pt, Coimbra, Portugal \\ ${ }^{4}$ Nextworks, g.landi@nextworks.it, Piza, Italy \\ ${ }^{5}$ VTT Technical Research Centre of Finland, first.lastname@vtt.fi, Oulu, Finland
}

\begin{abstract}
The anticipated deployment of IEEE 802.16-based wireless metropolitan area networks (WMANs) will usher a new era in broadband wireless communications. The adoption of the WiMAX technology for remote areas, for example, can address challenging scenarios in a cost-effective manner. While WiMAX Forum documents describe an architecture that inherently supports Quality of Service and mobility, several areas are left uncovered. We present an architecture which integrates WiMAX, Quality of Service and mobility management frameworks over heterogeneous networks, developing mechanisms for seamless handovers. Our approach takes into consideration the expected deployment of, on the one hand, the IEEE 802.21 (Media Independent Handover) proposed standard and, on the other, the IETFstandardized Next Steps in Signalling framework. The first contributions of this paper comprise a specification of the mechanisms for make-before-break vertical handovers taking Quality of Service signalling into account and the integration in a heterogeneous environment. The latter contribution is an empirical evaluation of the proposed architecture using a testbed demonstrator. We quantify the processing delays of the main components in our prototype implementation when a terminal hands over between different access technologies, demonstrating the potential of the proposed architecture.
\end{abstract}

\section{Introduction}

Broadband Wireless Access (BWA) technologies are expected to play a central role in Next Generation Networks (NGN) [1]. WiMAX [2], based on the IEEE 802.16 family of standards [3] [4], is one such technology that can form the foundation upon which operators can deliver ubiquitous Internet access in the near future. Operators care about making the most out of existing and future infrastructure expenditures. Of central concern in the emerging telecommunications environment is delivering seamless mobility while taking advantage of the different access networks, some of them already deployed, others, such as WiMAX, soon to be available. There are several proposals for fast and seamless mobility management between different access networks. IEEE has been working on the 802.21 draft standard [5] which enables Media Independent Handovers (MIH). IEEE 802.21 defines an abstract framework which delivers link layer information to the higher layers, in an effort to optimize heterogeneous handovers. When IEEE 802.21 is deployed, mobility management processes will be harmonized, irrespective of the underlying technology, considering that proper communication and interfaces are presented to the link layers.

Although the work within IEEE 802.21 is already in an advanced stage, the framework needs to be integrated with specific technologies, since each one has its specific mobility control procedures. Moreover, seamless mobility requires the active support of QoSrelated mechanisms in the handover process, guaranteeing that resources are reserved in the target access network before mobility management operations are completed. In other words, we cannot dissociate mobility management and QoS processes. We propose an architecture based on IEEE 802.21, which integrates the two mechanisms, and we empirically evaluate it using a real WiMAX testbed.

The aim of this paper is three-fold. First, we define a mobility architecture, based on IEEE 802.21, which supports seamless mobility in BWA networks, integrates different technologies, such as WiMAX and Wi-Fi, and is suitable for NGN environments. Second, 
we show how the proposed mobility architecture integrates QoS functionalities, specifying mechanisms to enable the complete combination of mobility and QoS, through the Next Steps in Signaling (NSIS) framework protocols [6] [7] [8]. Finally, we present an empirical evaluation of the proposed architecture. Using a real demonstrator, we report processing time for each module involved in handovers where WiMAX backhauls data.

This paper is organized as follows. Section II presents related work on mobility, QoS architectures and experimental testbeds. Section III introduces our mobility-QoS integrated architecture, its elements and functionalities. Section IV briefly describes how this architecture was implemented and section $\mathrm{V}$ presents our testbed, the performed tests and the obtained results. Finally, section VI concludes the paper and lists items left for future study.

\section{Related Work \& Background}

Due to the relevance of seamless mobility in future networks, a significant amount of related work has been published by the academic community until this moment. In [9] and [10], vertical handover schemes based on IEEE 802.21 are presented. Nevertheless, both proposals lack interaction between the MIH framework and the link layer access technologies QoS specificities. Furthermore, performance measurements are not given. In [11], a vertical handover scheme between UMTS and WiMAX, employing the 802.21 framework, is proposed. To guarantee service continuity, the authors define a new message for the IEEE 802.21 framework, which supports passive reservations during the $\mathrm{HO}$ preparation phase. However, resource activation is performed only after the physical handover is executed, delaying the packet delivery to the target access technology. Finally, in [12], a seamless mobility mechanism for heterogeneous environments is proposed. Instead of triggering events only from the MAC/PHY layers, the authors enhance the MIH platform with the capability to trigger events from the application layer as well, delivering this information to the mobility decision engine.

Up to now and to the best of our knowledge, there are very few implementations of the IEEE 802.21 framework in real testbed deployments. Nevertheless, the trends are changing and both manufacturers and standardization bodies are adopting uniform solutions to address inter-technology handovers. For example, Intel has recently demonstrated a basic seamless mobility solution between WiMAX and Wi-Fi using IEEE 802.21, as reported in [13], and both 3GPP and WiMAX Forum standardization bodies have also started to evaluate the impact of integrating IEEE 802.21 within their architectures.

In what concerns European funded projects, the Ambient Networks [14] has defined a novel triggerbased architecture for handover optimization [15], although not compliant with IEEE 802.21 framework. Interesting results are presented, demonstrating a handover between Ethernet and Wi-Fi. One of the well-known IEEE 802.21 deployments has been made in the European DAIDALOS project [16], which is addressing seamless mobility in heterogeneous environments. In this case, the IEEE 802.21 platform is considered as the means to implement protocol operations for seamless handovers, and further extended to support QoS provisioning along heterogeneous access networks [17]. However, results are yet to be presented that assess the feasibility and efficiency of the approach.

The integrated mobility and QoS architecture presented in this paper has been implemented in the European WEIRD project [18]. WEIRD is focused on WiMAX and proposes an architecture compliant with the most relevant standardization bodies, such as IEEE 802.16, IETF 16ng [19] and WiMAX Forum. In order to guarantee full interoperability among different WiMAX vendors, the WiMAX Network Reference Model (NRM) is used as a foundation, and the NSIS framework is adopted for QoS reservations. IEEE 802.21 is also considered and integrated into the WEIRD architecture to optimize mobility procedures. In order to demonstrate the feasibility of the proposed solution, the project has also developed a joint prototype which is deployed on four testbeds distributed across Europe (Finland, Italy, Portugal and Romania) and interconnected via the GEANT network.

As in DAIDALOS, WEIRD also uses the NSIS protocol for network layer QoS signaling. Nonetheless, WEIRD has extended the generic NSIS signaling layer to include specific WiMAX QoS parameters [20] [21]. Furthermore, a Media Independent Handover NSIS Signaling Layer Protocol (MIH NSLP) has been defined to transport the IEEE 802.21 MIH protocol messages across the network elements [22].

\section{Mobility Management in WiMAX}

This section presents the defined architecture for WiMAX, supporting and integrating mobility and QoS mechanisms. Subsequently, it provides a practical use case of the defined architecture, illustrating an intertechnology handover scenario. 


\subsection{QoS and Mobility Architecture}

The WiMAX Forum aims to define an end-to-end IP framework, with full interoperability between Base Stations (BSs) and Mobile Stations (MSs) from different vendors. WiMAX Forum thus extends the IEEE $802.16 \mathrm{~d} / \mathrm{e}$ architecture by defining a Network Reference Model (NRM). The NRM, illustrated in Figure 1, is a logical representation of the WiMAX network architecture, based on a set of functional entities and standardized interfaces, also known as reference points $(\mathrm{R} 1-\mathrm{R} 8)$. Three functional entities are defined: Connectivity Service Network (CSN), Access Service Network (ASN) and the Mobile Station (MS).

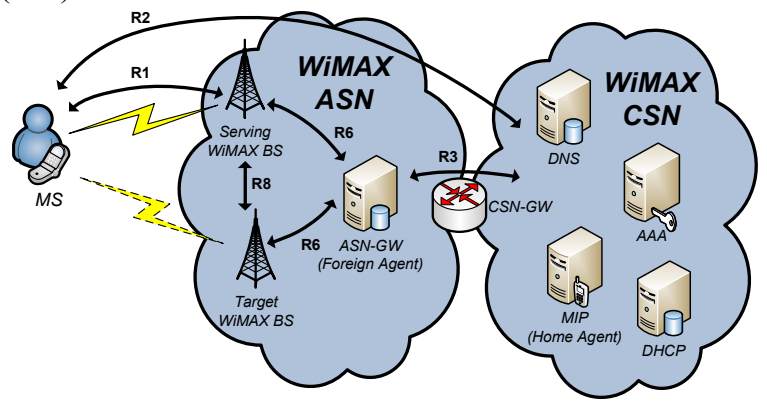

Figure 1: WiMAX Network Reference Model

The MS is responsible for establishing radio connectivity with the serving BS. The ASN is composed by a set of BSs connected to one or several ASN-Gateways (ASN-GW). The ASN-GW is the gateway for the ASN, establishing connectivity with the CSN. The ASN includes the required functionalities to provide radio connectivity with WiMAX subscribers, such as the establishment of signaling and data service flows (with the required WiMAX QoS parameters) in the WiMAX air link, as well as micro and macro mobility support. Additionally, it also performs relay functions to the CSN in order to establish IP connectivity and Authentication, Authorization and Accounting (AAA) mechanisms. The CSN provides connectivity with the IP backbone and holds DHCP, DNS and AAA servers, as well as Application Functions (AF) acting as the application-level controller.

The proposed mobility and QoS architecture, illustrated in Figure 2, is compliant with the WiMAX Network Reference Model (NRM), and thus composed by the CSN, ASN and MS. Quality of Service and mobility functionalities are managed in a coordinated way at the control plane level through the intercommunication and the combined processing of the Connectivity Service Controller (CSC) modules, located in each segment of the Network Reference Model (NRM).

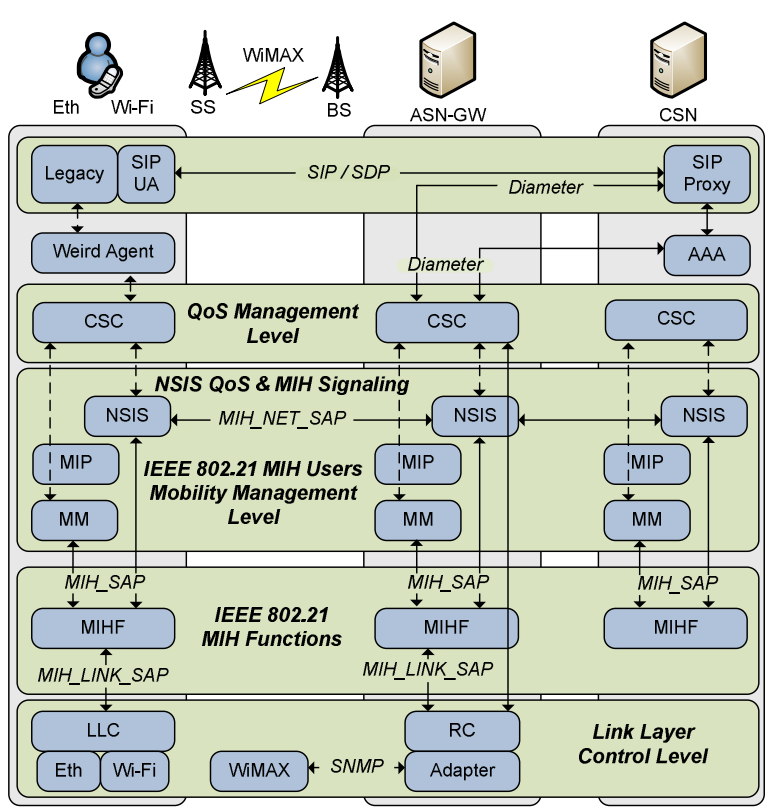

Figure 2: WEIRD mobility and QoS integrated architecture

During the session setup phase, the Connectivity Service Controllers (CSCs) interact with the service layer in order to retrieve information from the applications, regarding the traffic type and the required QoS parameters. In particular, two different approaches can be adopted in order to support both legacy and IP Multimedia Subsystem (IMS) applications, based on the Session Initiation Protocol (SIP) [23] and Session Description Protocol (SDP) signaling. For legacy applications, the QoS signaling is initiated by the MS. The Connectivity Service Controller located at the MS (CSC_MS) communicates with a module, called WEIRD Agent (WA), in charge of obtaining the application QoS parameters, such as required bandwidth, maximum latency and jitter. CSC_MS coordinates end-to-end QoS signaling, using the NSIS framework, translating the application QoS parameters to a QSPEC (Qos SPECification) adopted in the WiMAX NSIS model [24] and initiating the endto-end signaling towards the ASN, the CSN and the core network. In the case of IMS-like applications, the QoS signaling follows the network-initiated approach and it is strictly connected to the application layer SIP/SDP signaling. The SIP Proxy located at the CSN intercepts the SIP signaling between the SIP User Agents and extracts the session description from the SDP messages. The QoS parameters are forwarded to the CSC located at the ASN (CSC_ASN), through a set of Diameter [25] messages describing the media flows included in the sessions, where they are translated into WiMAX parameters. In this case the QoS NSIS 
signaling follows the edge-to-edge model since it is initiated and controlled by the CSC_ASN.

For both legacy and IMS-like applications, WiMAX resource reservations are handled by the ASN-GW through the interaction of the CSC ASN with the link layer level. In particular, the WiMAX Resource Control (RC) module hides all the WiMAX technology related functionalities from the higher layer entities of the architecture. It manages the WiMAX Service Flows (SFs) creation, modification and deletion, admission control mechanisms, and QoS policies enforcement on the WiMAX system through a set of technology dependent adapters. Detailed information about standalone WEIRD QoS management procedures has been published in [20] [21].

With respect to mobility procedures, the proposed architecture is based on the IEEE 802.21 [5] framework and on the standardized Mobile IP (MIP) [26] protocol. IEEE 802.21 introduces a new entity called MIH Function (MIHF), which hides the specificities of different link layer technologies from the higher layer mobility entities. Several higher layer entities, known as MIH Users (MIHUs), can take advantage of the MIH framework, including mobility management protocols, such as Mobile IPv4 (MIPv4) [26], Fast Mobile IPv6 (FMIPv6), Proxy Mobile IPv6 (PMIPv6) and SIP [23], as well as other mobility decision algorithms. In order to detect, prepare and execute handovers, the MIH platform provides three services: Media Independent Event Service (MIES), Media Independent Command Service (MICS) and Media Independent Information Service (MIIS). MIES provides event reporting such as dynamic changes in link conditions, link status and link quality. Since multiple higher layer entities may be interested in these events simultaneously, they may need to be sent to multiple destinations. MICS enables MIHUs to control the physical, data link and logical link layer. The higher layers may utilize MICS to determine the status of links and/or control a multimode terminal. Furthermore, MICS may also enable MIHUs to facilitate optional handover policies. Events and/or commands can be sent to MIHUs within the same protocol stack (local) or to MIHUs located in a peer entity (remote). Finally, MIIS provides a framework by which a MIHF located at the MS or at the network side may discover and obtain network information within a geographical area to facilitate handovers. The objective is to acquire a global view of all the heterogeneous networks in the area in order to optimize seamless handovers when roaming across these networks. Figure 3 illustrates the 802.21 MIH framework.

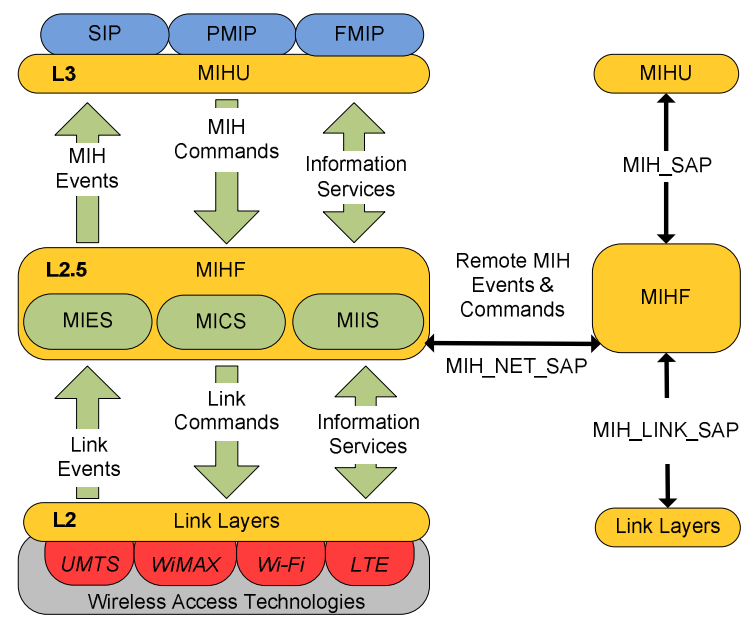

Figure 3: IEEE 802.21 MIH framework

In the WEIRD architecture (see Figure 2), the mobility management framework includes several instances of the MIHF, located at each segment of the WiMAX Network Reference Model. The MIH events are originated by the LLC (Link Layer Client) and include information about the link layer, such as the respective link status. The MIH commands are triggered by the MIHUs and are used to convey the handover decisions. The transport of the MIH protocol messages between remote MIHF peers is supported by the NSIS framework through the Media Independent Handover NSLP (MIH NSLP) [22]. The MIH NSLP was developed as an extension to the NSIS framework in order to transport the MIH protocol messages. There are two main reasons to sustain this approach. First, the IEEE 802.21 proposed standard does not specify any protocol for message exchange, providing only the requirements for such protocol, namely, security and reliability. Second, QoS signaling, which is tightly coupled with mobility, is performed through the QoSNSLP. In this context, the use of the NSIS framework to support both QoS and mobility processes, illustrated in Figure 4, becomes the natural choice, since it fulfills the requirements for MIH message exchange between remote entities.

The mobility management architecture includes a Mobility Manager (MM) instance, acting as a MIH User and strictly connected with the related Connectivity Service Controller (CSC), located on each segment of the Network Reference Model (NRM), and a Link Layer Client (LLC) located on the MS. The LLC is in charge of monitoring the link condition (signal level for Wi-Fi and WiMAX links, connected/disconnected cable for Ethernet). In case of link status variation, the related MIH event is triggered and sent to the local MIHF through the MIH_LINK_SAP. Here, it is delivered to the registered 
MIHUs, both local and remote MMs, through the MIH_SAP.

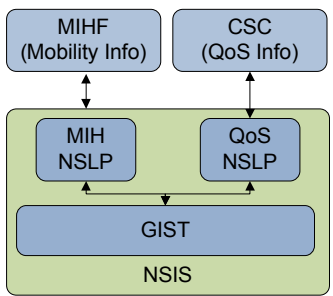

Figure 4: NSIS functional decomposition (QoS and MIH NSLPs)

The MIH events are used by the MM to update their internal status and detect new imminent handovers. In this case, the MM located at the MS searches for the availability of new target networks and requests a new resource reservation to the associated CSC. The entire procedure is performed jointly by the CSC and the MM: while the MM manages the link status and is able to take decisions about the handover executions, the CSC handles the sessions at the control plane and controls the resources for the associated traffic flows. Following the approaches used for resource control in the session setup phase, the handover procedure and the wireless link reconfiguration are controlled by the MM located at the MS (MM_MS) for host-initiated sessions and by the MM located at the ASN (MM_ASN) for IMS-like applications. When the MS moves between different ASNs, the entire procedure is controlled by the MM located at the CSN (MM_CSN), which takes the final handover decision. However, the actual resource reservation is still performed by the $\mathrm{CSC}$ at the ASNGW (CSC_ASN). Handover decisions are finally notified to the lower layers using the MIH commands delivered to the LLC.

\subsection{A Practical Use Case}

Up to now we have described the mobility and QoS architecture modules and their operation. In the following paragraphs we will present a practical use case of an inter-technology handover involving WiMAX as the backhaul access technology, demonstrating efficient management of control plane functionalities, as well as data plane configuration and QoS resources reservation. The example scenario is shown in Figure 5. It consists of a MS with two network interfaces (Ethernet and Wi-Fi), initially connected to an Ethernet cable, backhauled by a WiMAX fixed Subscriber Station (SS) (serving SS). Later on, the user decides to move away from his desk and unplugs the Ethernet cable. Consequently, the MS connects to the Wi-Fi network, backhauled by another
WiMAX fixed SS (target SS), located in the same ASN of the serving SS. This type of scenario includes interand intra-technology mobility procedures: the MS is connected via Ethernet and makes an inter-technology handover to a Wi-Fi network; at the same time, there is an intra-technology handover from the serving WiMAX SS to the target WiMAX SS in the backhaul, following the intra-ASN WiMAX mobility model.

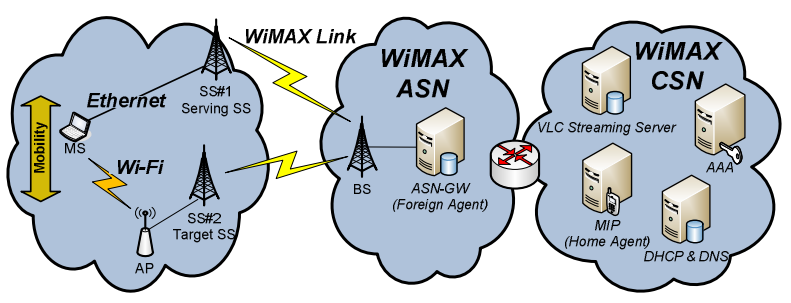

Figure 5: Deployed scenario

Figure 6 illustrates the seamless handover signaling diagram between Ethernet and Wi-Fi, backhauled by WiMAX. After connecting the terminal, two Link_Up events are sent by the LLC to notify the MIHF that Wi$\mathrm{Fi}$ and Ethernet networks are available. The MIHF forwards these events to the registered MMs (local and remote) (step 1). As a result, the MMs update their internal state machine with the new available access technologies.

When the user starts a legacy application, the resource reservation procedure is triggered by the WEIRD Agent (WA) and the end-to-end QoS NSIS signaling is initiated [20] [21] (step 2). As a result, a set of WiMAX Service Flows (SFs) are created by the $\mathrm{RC}$ between the serving SS (SS\#1) and the WiMAX $\mathrm{BS}$ in order to assure the required QoS (step 3).

Thereafter the user interacts with the LLC in order to unplug the Ethernet cable and move to the Wi-Fi link. The LLC detects that the Ethernet connection is going down and sends a Link_Going_Down event to the MIHF located at the MS that forwards it to the registered MMs (step 4). The MM MS internal state machine is updated again and, since the Ethernet link is going down, triggers the Handover Preparation phase to reserve the new resources in the target link before the Ethernet cable is unpluged. The MM_MS selects the Wi-Fi link as the target network for the handover according to the current status of the internal machine and notifies this decision to the CSC_MS. Here, a new NSIS QoS signaling is triggered (step 5) to update the resource reservation for the existing sessions and create new Service Flows (step 6) in the target network segment (composed by WiMAX and Wi-Fi). The NSIS response message notifies the CSC_MS that resources have been allocated between the target SS (SS\#2) and BS and that they can be used 
by the data traffic flows after the handover. At this point the MS can move from the home network to the foreign network where it will be able to maintain the same QoS level.

Since the composed target access network is already prepared to receive the MS, the MM sends a Link_Action command to the LLC in order to start the handover execution phase (step 7). During the Handover Execution phase the user unplugs the Ethernet cable from the MS, the Wi-Fi network interface starts the MIP registration procedures with the FA, and the MIP tunnel between the FA and the $\mathrm{HA}$ at the ASN is established. Data traffic is carried through the Wi-Fi link and is mapped to the new WiMAX SFs between the target SS and BS on the WiMAX link, assuring the QoS level originally required by the active applications.

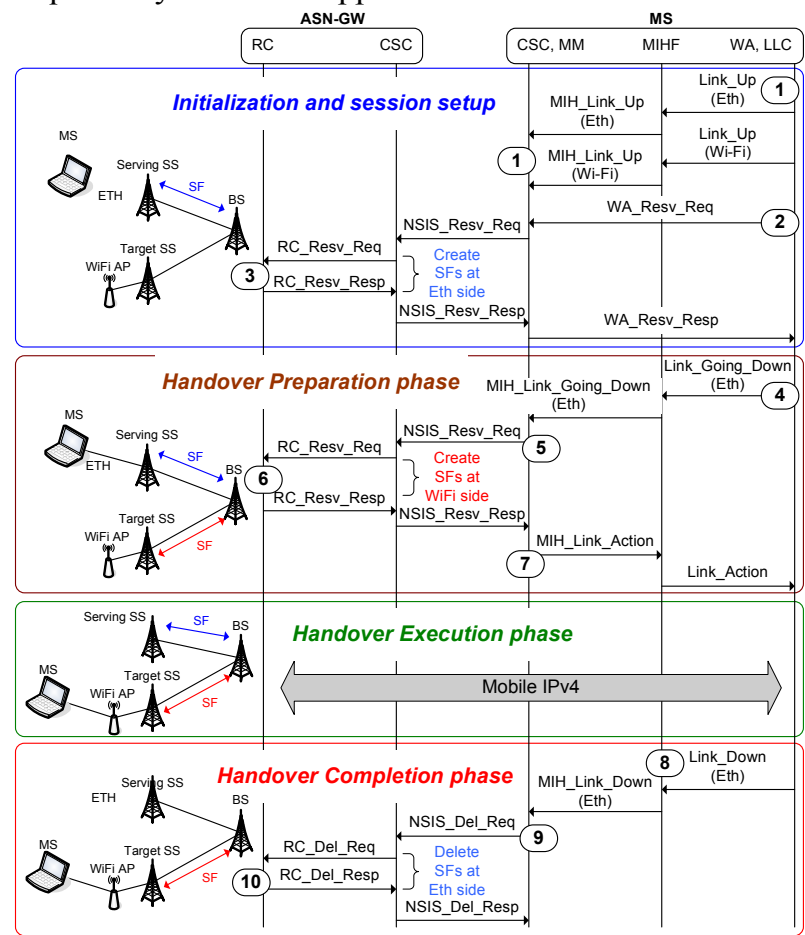

Figure 6: Signaling diagram for QoS-aware handover

The resources previously allocated between the serving SS and BS are released during the Handover Completion phase. When the Ethernet cable is unplugged, the LLC sends a Link_Down event (step 8), forwarded by the MIHF to the MM_MS. The CSC_MS, as responsible for the dynamic control of sessions and resources, is in charge of handling the deletion of the old WiMAX Service Flows for the existing sessions and initiates the related NSIS QoS signaling towards the CSC_ASN (step 9). Finally, the
RC deletes the SFs in the previous WiMAX link (step 10).

\section{Implementation}

This section briefly describes the implementation of the main mobility modules, namely NSIS, LLC, MIHF and MM.

\subsection{NSIS}

NSIS, as a framework for QoS signaling, decouples the transport layer from the signaling layer. In the NSIS framework, GIST provides the transport and association mechanisms necessary for QoS signaling. QoS NSLP instructs GIST on the NSIS nodes to signal in order to guarantee the applications QoS requirements. MIH NSLP enables the transport of MIH messages between MIH peers. Both QoS NSLP and GIST are conformant with the specifications of the IETF NSIS working group [6] [7] [8], whilst the MIH NSLP was included in the WEIRD architecture to transport MIH messages between peer remote entities [22]. The MIH NSLP module has a northbound interface with MIHF, compliant with the MIH_NET_SAP specified in the IEEE 802.21 standard, and a southbound interface with GIST acting according to the specification of GIST (see Figure 4). For MIH events/commands propagation, MIHF delivers the messages to the MIH NSLP. The MIH NSLP parses the received message and creates the necessary information to instruct GIST on the delivery process. Such information includes the MIH message and Message Routing Information (MRI) which contains information such as the type of transport required (e.g. TCP for reliable delivery). The NSIS framework related modules have been implemented in the Java programming language.

\subsection{LLC}

The aim of the Link Layer Client (LLC) was to implement a link information collector independent of the specific hardware, vendor, or GNU/Linux kernel. For this, Linux natively provides convenient ways for application layer software to gather link specific information from the kernel and directly from the network device drivers without modifications to both of them. LLC constantly monitors the network link states and, based on this information, provides events through an Event Trigger module to the registered MIHF. For simplicity, in the examined scenario, LLC provides only Link_Up, Link_Down and synthetically generated Link_Going_Down events. The monitored link types are Ethernet and Wi-Fi. 
Link states are identified in the Generic Link State Monitor (GLSM) by observing the operation status of access network interfaces. After each link is operationally up and its link type has been identified, GLSM initiates the Link-specific Information Monitor (LSIM) which acquires link-specific information. For instance, LSIM can obtain Access Point (AP) information for Wi-Fi accesses. This information is gathered using ioctl system calls.

\subsection{MIHF}

The MIHF is the core entity of the IEEE 802.21 framework. It provides communication with lower layers through MIH_LINK_SAP, with upper layers through MIH SAP and with remote MIHFs via MIH_NET_SAP, using the MIH protocol [5]. During initialization, each MIHF must be configured and thereafter it automatically creates the communication sockets for each one of the standardized interfaces. Maps of events, commands and information services are associated with each one of the MIHFs. The MIH Users will also be associated to these sets of maps, after having subscribed to one (or more) of the MIH services (MIES, MICS and MIIS). The MIHF receives messages from the MIHU, LLC or remote MIHF and reacts accordingly. For example, after receiving a link event from the LLC though the MIH LINK SAP, the MIHF must look for the subscribed MIHUs to this event on the events list. For local MIHUs, the MIHF must generate the correspondent MIH event and send it through the MIH_SAP, whereas for remote MIHUs subscriptions, the MIHF must deliver the MIH event to the MIH NSLP through the MIH_NET_SAP.

\subsection{CSC \& MM}

As mentioned above, each segment of the WiMAX network is managed by a Connectivity Service Controller (CSC), with its own Mobility Manager (MM). CSC has, as its main role, to manage sessions at the control plane, coordinating all relevant related signalling at different layers and the resource reservation in the WiMAX link, which is dynamically updated during the session setup and the handover phases. In particular, the CSC_MS is the main coordinator for sessions of applications based on hostinitiated QoS signalling, while the CSC_ASN has the same role for IMS-like applications that adopt the network-initiated approach. Resource allocation on the WiMAX link follows the network-initiated model and is handled by the CSC_ASN at the ASN-GW.

The resource update for mobility follows the makebefore-break approach: when an imminent handover is detected, new SFs are allocated on the target segment, while resources on the old path are released at the end of the handover execution phase. These procedures are completely transparent for the application layer and are managed by the entity that acts as the main coordinator for the sessions involved in the handover: the MM_MS for host-initiated sessions and the MM_ASN for IMSlike applications.

Link layer information about the wireless link status is monitored by LLC and sent to the MM module through a set of MIH events carried by the MIH NSLP signalling (for remote events). The strong interaction between the CSC, which manages the sessions at the control plane, and the related MM, which manages the link layer MS status, allows the system to allocate new resources in the target link for the existing traffic flows whenever a new handover is detected through the Link Going Down event. Following the same approach, previously used resources are removed when the Link_Down message is received, as presented in Figure 6.

\section{Testbed Evaluation}

This section describes the empirical evaluation of the proposed mobility management architecture prototype. The experimental scenario is illustrated in Figure 5. The testbed includes modules that implement the CSN, ASN and MS functionalities. Under the ASN, a real, commercial-of-the-shelf (COTS) WiMAX BS is directly connected to the ASN-GW. Two WiMAX SSs are connected to the BS creating a Pointto-Multipoint topology. The MS is connected to SS\#1 by Ethernet and to SS\#2 by Wi-Fi. A streaming server is located in the CSN broadcasting a video towards the MS.

The goal of this scenario is to demonstrate a handover process between Ethernet and Wi-Fi, backhauled by a fixed WiMAX link, while the MS is receiving a video stream. Initially the MS is connected to SS\#1 using an Ethernet cable. For the video stream to traverse the WiMAX link towards the MS it is required to establish two Service Flows between the BS and SS\#1, one for the downlink and one for the uplink, with $512 \mathrm{Kbps}$ each. While the user is receiving the video stream through the concatenated WiMAX and Ethernet link, he decides to unplug the Ethernet cable and connect to the Wi-Fi link. This behavior automatically triggers a vertical handover procedure from Ethernet to Wi-Fi, leading to the handover preparation phase. During this phase, the required resources on the concatenated WiMAX (BS and SS\#2) and Wi-Fi link are reserved for the MS. After the preparation phase is complete, the user executes the physical handover to $\mathrm{Wi}-\mathrm{Fi}$ and resumes the video stream. 
The performance of the proposed mobility architecture is addressed in this section. The internal processing times of the several modules involved during the different handover phases are analyzed. Additionally, the performance of the MIH transport mechanism for the communication between peer IEEE 802.21 entities is also evaluated.

\subsection{Handover Processing Time}

As discussed in Section 3, the handover procedure follows the make-before-break model and consists of three main sequential phases: preparation, execution and completion. The preparation phase includes all the required procedures to configure the target network, whereas during the handover execution phase the data path towards the foreign network is established. Finally, existing resources on the old path are released during the handover completion phase.

Figure 7 shows the processing and communication time for handover preparation and completion phases, $736 \mathrm{~ms}$ and $655 \mathrm{~ms}$, respectively. In both cases the most time consuming component is the NSIS bidirectional communication between the MS and the ASN-GW (87\% for handover preparation and 94\% for handover conclusion). This is due to the NSIS message association performed by GIST between the first nodes on the preparation phase. Additionally, all the signaling between the MS and ASN nodes crosses the WiMAX link, with approximately $30 \mathrm{~ms}$ of delay.

The handover execution time, measured as the time interval between the instant when the Ethernet interface stops receiving the video stream and the moment when the Wi-Fi interface resumes the stream, is approximately $4199 \mathrm{~ms}$. The high value of the handover execution time is due to the MIPv4 protocol, specifically, because of the inherent latency problems caused by the packet tunneling between the MIP Home Agent (HA) and Foreign Agent (FA). FMIPv6 protocol has major improvements relatively to MIPv4, including redundancy of Foreign Agent entities in the network, a native solution to avoid the triangle routing, dynamic configuration for care-of-addresses and improved security. Nevertheless, to avoid adding additional complexity to the demonstrator, MIPv4 has been used to handle IP mobility management. Furthermore, the testbed aim is to evaluate the effective management and coordination of the proposed mobility and QoS architecture during an inter-tech handover process, and not to evaluate the performance of the IP mobility management protocol.

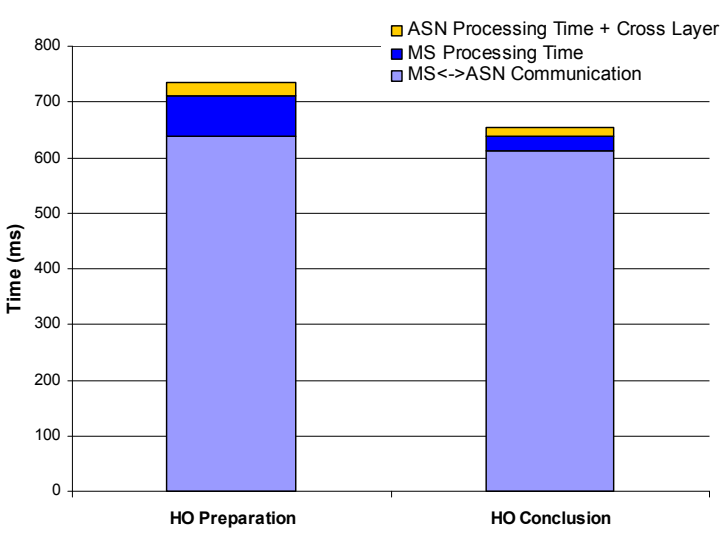

Figure 7: Processing and communication time for HO preparation and completion phases

To study the individual behavior of the MS and ASN-GW entities, Figure 8 illustrates the processing time for each one of the internal modules of these entities during the handover preparation and completion phases.

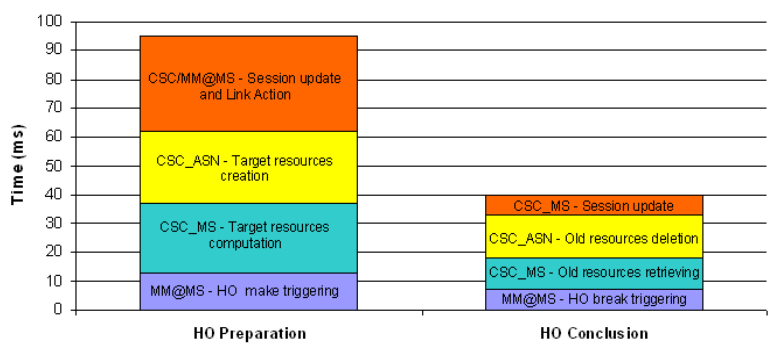

Figure 8: Internal modules processing time for HO preparation and completion phases

The MS processing time is approximately $70 \mathrm{~ms}$ for the handover preparation and $25 \mathrm{~ms}$ for the handover completion. Initially, for the handover preparation, the MM_MS updates the internal state machine with the new status of the MS Ethernet connection and triggers the handover. Thereafter, the CSC_MS retrieves the QoS requirements of the stored sessions and computes the new resources to be allocated in the target link for each one of them. The corresponding NSIS QSPEC is sent to the NSIS module to initiate the signaling to the CSC ASN through the WiMAX link. The processing time for this first step is approximately $37 \mathrm{~ms}(13 \mathrm{~ms}$ for the MM_MS and $24 \mathrm{~ms}$ for the CSC_MS). After the resources reservation in the WiMAX BS are completed, the CSC_MS updates the status of the stored sessions, and the MM MS sends the Link_Action message to the MIHF module, with a total processing time of approximately $33 \mathrm{~ms}$. 
The processing time at the ASN-GW, including both the processing of the CSC_ASN and the RC, is approximately $25 \mathrm{~ms}$ for the handover preparation and $15 \mathrm{~ms}$ for the handover completion. These modules do not take any active handover decision, since they are only in charge of the WiMAX resources reconfiguration for the active sessions, as specified in the received QSPEC. In particular, during the handover preparation phase the new Service Flows are allocated and activated in the WiMAX segment towards the target SS, while during the handover completion the Service Flows are deleted over the serving WiMAX link.

With respect to the MIHF, during the handover preparation phase, the processing time to forward the Link_Going_Down event received from the LLC is nearly $215 \mu \mathrm{s}$ (as illustrated in Figure 9). After receiving the Link_Action from the MM_MS and before sending the message to the LLC, the MIHF processing time is approximately $145 \mu \mathrm{s}$. During the handover completion phase, the MIHF takes about 215 $\mu$ s to process the Link Down event received from the LLC. It is noticeable that the internal processing time of the MIHF is much smaller than in the CSC modules. The MIHF, when properly configured and initialized, just has to forward events and commands to the MIHUs and LLCs. Finally, the communication time between the MIHF and the CSC_MS is around $750 \mu \mathrm{s}$ for each direction.

\subsection{MIH Transport Mechanisms}

As stated before, the MIH transport mechanism relies on the NSIS communication facilities, namely GIST, to assure the transport of messages, and MIH NSLP to instruct GIST on the delivery process. The processing time of GIST includes the parsing of MIH messages received from the MIH NSLP, the forwarding to the next peer, and the refresh mechanisms to keep the associations. Since the MS acts as the initiator, GIST has a higher processing time when compared with the ASN. This observation is due to the decision process on the transport protocol (UDP or TCP), as well as on the message association mechanism required by GIST. On the ASN side, the GIST processing time is nearly $7.5 \mathrm{~ms}$.

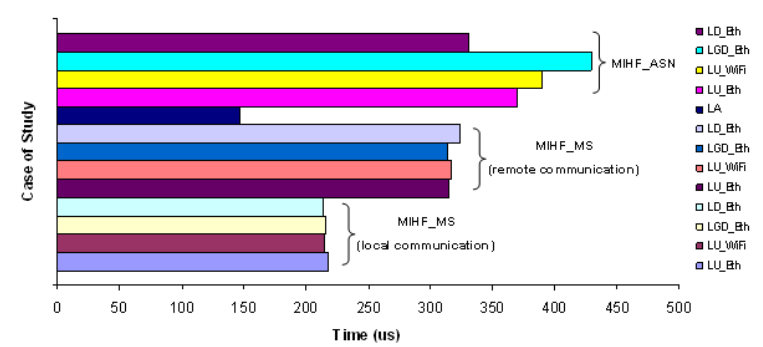

Figure 9: MIHF processing time

At the MS, the MIH NSLP processes the messages received by the MIHF (MIH messages to be transported to a remote MIHF), and due to the messages received, the MIH NSLP instructs GIST on the delivery process through the MRI serialization. The MIH processing at the MS takes approximately $25 \mathrm{~ms}$ and includes the parsing of MIH messages in order to map the destination ID to an IP address (required by the forwarding process of GIST). The MRI Serialization at the MS side is nearly $15 \mathrm{~ms}$. At the ASN side, the MIH NSLP handles the messages received from GIST and performs the necessary processing to deliver the MIH messages to the remote MIHF. This process takes around $7.5 \mathrm{~ms}$. All these values are small and do not compromise the handover efficiency.

In a remote communication, when the MIHF forwards the events sent by LLC, the MIHF processing time is nearly $310 \mu \mathrm{s}$. Then, when the MIHF at the ASN receives the MIH messages from NSIS, it has a processing time of nearly $300 / 400 \mu \mathrm{s}$, in order to forward them to CSC.

\section{Conclusions \& Future Work}

As mobile communication becomes widespread over a broad set of wireless technologies, there is the need for mechanisms that support seamless intertechnology handover. Moreover, given the users and next generation applications requirements, intertechnology handover mechanisms must be developed while maintaining the adequate levels of quality of service. This paper has described an architecture for mobility management in WiMAX networks.

Mobility management in heterogeneous environments, with inter-technology handovers, can be substantially improved by the use of a unifying framework such as the Media Independent Handover described in IEEE 802.21. With such an approach, the details of the underlying technologies become transparent to the upper layers, allowing a smoother support of vertical handovers. Seamless handovers need a make-before-break solution, where resources are reserved in the target network before the 
connection to the serving network is broken. In the described mobility management architecture, the Media Independent Handover proposed standard was associated with the Next Steps in Signalling framework for achieving quality of service signalling in the inter-technology mobility scenario.

This paper described the WiMAX architecture, with emphasis on the mobility management mechanisms and on the Media Independent Handover standard. Then, the quality of service aware mobility management architecture to support seamless handovers was presented in association with the use case developed in the context of the WEIRD project. Implementation aspects about the integration of the Media Independent Handover standard with the Next Steps in Signalling framework within the mobility management architecture were also detailed. Finally, the performance of the main mobility and Quality of Service modules was evaluated on a testbed and the results obtained were discussed.

As future work, we plan to integrate the architecture with a fast mobility protocol, as well as extend it for other access technologies, such as 3GPP UMTS/LTE and DVB, and support inter-ASN handovers.

\section{References}

[1] ITU-T, General Principles and General Reference Model for Next Generation Networks, Rec. Y2011, Oct. 2004.

[2] WiMAX Forum, WiMAX End-to-End Network Systems Architecture Stage 2-3: Architecture Tenets, Reference Model and Reference Points, Release 1, Jan. 2008.

[3] IEEE 802.16 WG, IEEE Standard for Local and Metropolitan Area Networks. Part 16: Air Interface for Fixed Broadband Wireless Access Systems, IEEE Std. 802.162004, Oct. 2004.

[4] IEEE 802.16 WG, Amendment to IEEE Standard for Local and Metropolitan Area Networks. Part 16: Air Interface for Fixed Broadband Wireless Access Systems Physical and Medium Access Control Layer for Combined Fixed and Mobile Operation in Licensed Bands, IEEE Std. 802.16e-2005, Dec. 2005.

[5] IEEE 802.21 WG, IEEE Draft Standard for Local and Metropolitan Area Networks: Media Independent Handover Services, IEEE P802.21/D10.0, April 2008.

[6] R. Hancock et al, Next Steps in Signaling (NSIS): Framework, IETF RFC 4080, June 2005.

[7] H. Schulzrinne, R. Hancock, GIST: General Internet Signalling Transport, IETF NSIS WG Internet-Draft, July 2008.

[8] J. Manner, G. Karagiannis, NSLP for Quality-of-Service Signaling, IETF NSIS WG Internet-Draft, Feb. 2008.
[9] L. Eastwood et al., Mobility Using IEEE 802.21 in a Heterogeneous IEEE 802.16/802.11-Based, IMT-Advanced (4G) Network, IEEE Wireless Communications Magazine, pp. 26-34, April 2008.

[10] G. Lampropoulos et al., Media Independent Handover for Seamless Service Provision in Heterogeneous Networks, IEEE Communications Magazine, pp. 64-71, Jan. 2008.

[11] J. Baek et al., Network Initiated Handover Based on IEEE 802.21 Framework for QoS Service Continuity in UMTS/802.16e Networks, in Proc. IEEE Vehicular Technology Conference, VTC Spring 2008, pp. 2157-2161, May 2008.

[12] W. Ying et al., An Enhanced Media Independent Handover Framework for Heterogeneous Networks, in Proc. IEEE Vehicular Technology Conference, VTC Spring 2008, pp. 2306-2310, May 2008.

[13] Intel Wi-Fi to WiMAX seamless handover demonstration

http://blogs.intel.com/research/2008/02/wifi_wimax handov er.php

[14] The Ambient Networks project - http://www.ambientnetworks.org/

[15] K. Pentikousis et al., The Ambient Networks Heterogeneous Access Selection Architecture, in Proc. First Ambient Networks Workshop on Mobility, Multiaccess, and Network Management (M2NM), Sydney, Australia, Oct. 2007, pp. 49-54.

[16] The DAIDALOS project - http://www.ist-daidalos.org

[17] S. Sargento et al, Integration of Mobility and QoS in 4G Scenarios, in Proc. Third ACM Workshop on QoS and Security for Wireless and Mobile Networks, Chania, Crete Island, Greece, Oct. 2007, pp. 47-54.

[18] The WEIRD project - http://www.ist.weird.eu

[19] S. Madanapalli, Analysis of IPv6 Link Models for IEEE 802.16 Based Networks, IETF RFC 4968, Aug. 2007.

[20] P. Neves et al, A Vendor-Independent Resource Control Framework for WiMAX, in Proc. 13th IEEE Symposium on Computers and Communications (ISCC), Marrakech, Morocco, July 2008, pp. 899-906.

[21] B. Sousa et al, The Cost of Using IEEE 802.16 Dynamic Channel Configuration, in Proc. IEEE International Conference on Communications (ICC), Beijing, China, May 2008, pp. $383-390$.

[22] L. Cordeiro et al, Media Independent Handover Network Signalling Layer Protocol (MIH NSLP), IETF NSIS WG Internet-Draft, Feb. 2008.

[23] J. Rosenborg et al, SIP: Session Initiation Protocol, IETF RFC 3261, June 2002.

[24] N. Ciulli et al, A QoS model based on NSIS signalling applied to IEEE 802.16 networks, $2^{\text {nd }}$ IEEE International Broadband Wireless Access Workshop, Jan. 2008.

[25] P. Calhoun, J. Loughney, E. Guttman, G. Zorn, J. Arkko, Diameter Base Protocol, IETF RFC 3588, Sept. 2003. [26] C. Perkins, IP Mobility Support, IETF RFC 2002, Oct. 1996. 